\title{
Possibilities at the Polar beamline at APS
}

\section{Joerg Strempfer, Steven Kearney, Altaf Khan, Daniela Capatina, Deming Shu, Ruben Reininger, Luca Rebuffi, Yongseong Choi, Gilberto Fabbris, Daniel Haskel}

\author{
Argonne National Laboratory, Lemont, United States of America; \\ strempfer@anl.gov
}

In 2024, the upgrade of the Advanced Photon Source at Argonne National Laboratory to an MBA reverse bent lattice will be completed. APS-U will offer extremely brilliant and highly coherent beam through the new low emittance source [1] to the user community. This will enable a variety of exciting new possibilities for dichroic scattering and spectroscopy experiments by pushing towards extreme pressures and high spatial resolution. Polar, the beamline for polarization modulation spectroscopy at sector 4 of the APS will make use of these new possibilities in terms of small focus sizes, coherence and polarization.

Fast polarization flipping between left and right circular as well as between horizontal and vertical linear polarization will be possible with the new Superconducting Arbitrarily Polarizing Emitter (SCAPE) undulators which are currently being designed for the Polar beamline. Two in-line SCAPE undulators will produce horizontal and vertical linear polarization as well as left and right circular polarization in the energy range from 2.7 to $27 \mathrm{keV}$, thanks to the implementation of small diameter round ID vacuum chambers enabled by on-axis injection at APS-U. Two experimental setups will make use of this new source and will allow diffraction (XRD, REXS, XRMR) as well as absorption spectroscopy (XMCD, XMLD) experiments covering all relevant absorption edges.

The beamline will make accessible especially the energy range above $14 \mathrm{keV}$ for magnetic spectroscopy experiments, normally not reachable at conventional hard-x-ray beamlines using phase plates for polarization manipulation and will enable investigation of magnetic properties of materials at the $5 \mathrm{f} \mathrm{L-and} 4 \mathrm{~d}$ K-edges using spectroscopic methods. Small focused and coherent beams down to $100 \mathrm{~nm}$ will allow reaching new areas in terms of resolution, by employing direct imaging or ptychographic methods, at low temperature, high magnetic fields and high pressures. Beamline optics are designed to reduce vibrations to guarantee small focus sizes.

A low vibration, large bore superconducting magnet with $9 \mathrm{~T}$ longitudinal and $1 \mathrm{~T}$ transversal fields will allow XMCD and XMLD measurements at extreme pressures using the small beam focused by KB optics. A horizontal diffractometer with an optional $2 \mathrm{~T}$ superconducting magnet will allow dichroic diffraction and spectroscopy experiments in moderate fields and at high pressures. An interchangeable high-precision sample stage will allow for 3D dichroic imaging experiments using highly focused beam.

[1] https://www.aps.anl.gov/Beamline-Selection/Technical-Information/Storage-Ring-Parameters

Keywords: spectroscopy, polarization, coherence, diffraction 